\title{
Deconvolution Method for Directions of the Post-Depositional Detrital Remanent Magnetization Using an Exponential Fixing Function
}

\author{
Hideo TSUNAKAWA* \\ Institute of Research and Development, Tokai University, Hiratsuka, Kanagawa 259-12, Japan
}

(Received November 7, 1994; Revised May 8, 1995; Accepted June 3, 1995)

\begin{abstract}
A simple deconvolution method of the post-depositional DRM (detrital remanent magnetization) is proposed and examined using the synthetic data. Assuming the exponential fixing function of magnetic moments in sediments, the original field is expressed by a simple function of the convolved field. This leads us to the deconvolution of the post-depositional DRM if the fixing depth is known. In general the fixing depth is not given, so that the problem becomes complicated. However, adopting a few reasonable assumptions, the deconvolution is performed as a nonlinear problem with the fixing depth being an unknown parameter. Consequently the original field including the relative field intensity can be estimated from the direction data sets with the new method.
\end{abstract}

\section{Introduction}

The sediment continuously records the ancient geomagnetic field as the detrital remanent magnetization (DRM). This continuity is one of the important advantages for the palaeomagnetism in comparison with volcanic rocks. From the various laboratory experiments and observations, the DRM acquired after the deposition is important as well as the DRM acquired at the deposition (e.g. Irving and Major, 1964; Kent, 1973; Løvlie, 1974). The post-depositional DRM is generally originated from magnetic particles rotating in the interstices filled with water (Irving, 1957).

The DRM acquisition mechanism has been investigated for more than fifty years and Verosub (1977) gives a comprehensive review on the earlier studies. In the beginning of the studies on DRM mechanism, the depositional DRM is considered to play a main role in the acquisition of the sedimentary magnetism (e.g. Nagata, 1962). The problem of the depositional DRM is inaccuracy of the magnetization direction compared with the ambient field. There are a few kinds of the directional error: an inclination error, a bedding error, and an error due to the current rotation effect (e.g. King, 1955). These errors are sometimes found in glacial varve samples. However, it is concluded that the post-depositional DRM records the field direction almost accurately in accordance with the laboratory experiments and the palaeomagnetic measurement of deep-sea sediments (Verosub, 1977; Barton and McElhinny, 1979; Barton et al., 1980). Although the inclination error of natural and redepositional deep-sea sediments has been reported (e.g. Blow and Hamilton, 1978), it may be explained by mixing of the depositional and the post-depositional DRMs suggested by Verosub (1977) or by the effect of the gravitational compaction (e.g. Anson and Kodama, 1987; Arason and Levi, 1990).

Irving (1957) suggests that magnetic particles in void cannot rotate towards the ambient field direction if a water content is lower than a critical value. Verosub (1977) attempted to elucidate the natural state of the DRM by applying a constraint of the critical water content and by taking account of physical processes of the bioturbation and gravitational compaction. According to the experiment by Verosub et al. (1979), remagnetization after the deposition, namely the post-depositional DRM, takes place only for

*Present address: Department of Earth and Planetary Sciences, Tokyo Institute of Technology, Meguro-ku, Tokyo 152, Japan. 
a water content higher than a critical value, but stirring the sediment like the bioturbation can generate remagnetization of the sample. Barton and McElhinny (1979) and Barton et al. (1980) support this conclusion from their experiments. Whether a water content just after the deposition is lower than a critical value or not depends upon size, shape, composition, and mineralogy, in particular, upon the grain size distribution of matrices (Payne and Verosub, 1982).

The post-depositional DRM may result in modulating the ambient field variation. Løvlie (1974) explicitly pointed out the time lag of the post-depositional DRM from the external signal of the magnetic field. It is further suggested that the magnetization intensity decreases in the transition due to the convolution of the antipodal fields and thus the short event of the polarity reversal is possibly masked in the post-depositional DRM (Løvlie, 1976). For example, the deep-sea sediment often shows the intensity decrease of 1-2 order of magnitude near the polarity change (e.g. Opdyke et al., 1973). Niitsuma (1977) explained it by the zone magnetization model in which the post-depositional DRM is acquired within a certain thickness below the depositional surface. This mechanism results in the compensation of the antipodal magnetizations which were acquired in the geomagnetic field before and after the polarity reversal. Analysing several deep-sea cores, Niitsuma (1977) estimated the thickness to be about $40 \mathrm{~cm}$.

Bioturbation is often found in marine sediments. If the post-depositional DRM is acquired through the bioturbation, the magnetization intensity is related to the activity of burrowing organisms in the sediment. The previous investigation on the depth relationship of the biological activity in the sediment suggests that worms can burrow as deep as a half meter and the mean depth may be of the order of $10 \mathrm{~cm}$ (e.g. Guinasso and Schink, 1975).

Another observation of the fixing depth was made using ${ }^{10} \mathrm{Be}$ concentration in the marine sediment (Raisbeck et al., 1985). ${ }^{10} \mathrm{Be}$ is cosmogenic and is regarded as a possible indicator of the geomagnetic field intensity. A $10-15 \mathrm{~cm}$ depth lag between the changes in the ${ }^{10} \mathrm{Be}$ concentration and in the palaeomagnetic inclination was found at the Matuyama-Brunhes boundary. They concluded that the remanent magnetization of the sediments were locked at a depth of $10-15 \mathrm{~cm}$.

The sediments redeposited in the laboratory sometimes show that the amount of remagnetization gradually decreases in drying (Verosub et al., 1979; Barton et al., 1980; Payne and Verosub, 1982). The redeposition experiments of synthetic and natural sediments by Hamano (1980) indicated that magnetic particles are locked also during the compaction and result in the growth of the post-depositional DRM. In his experiment the compaction of sediments terminated at an overburden pressure of $0.5-1 \times 10^{5} \mathrm{~Pa}$. This effective pressure is exerted by the overlying sediment of 10-20 m thickness if the density contrast between the sediments and the sea water is $5 \times 10^{2} \mathrm{~kg} \mathrm{~m}^{-3}$. Similar results were obtained for various sediments by Deamer and Kodama (1990).

Yamazaki (1987) measured the physical properties for the siliceous clay sediment sampled by the box-corer from the Pacific Ocean. The results indicated the steep change in water content, porosity and density within the depth less than $10 \mathrm{~cm}$. The intensity of natural remanent magnetization increases up to twice there and becomes almost constant in the deeper part. Hence the fixing depth of magnetic particles seems to be about $10 \mathrm{~cm}$ for this sediment.

These results suggest that the fixing depth of magnetic particles in sediments is probably variable case by case. As the wide range of the fixing depth may be attributed to the biological environment and to the physical property of the sediment, it seems to be difficult to evaluate the effect of convolution on the post-depositional DRM only from the laboratory experiment. The theoretical formulation is, therefore, helpful for restoration of the original field from the post-depositional DRM. In this paper, a new deconvolution method of post-depositional DRM directions is proposed and its validity is checked using the synthetic data.

\section{Previous Formulation of the Post-Depositional DRM}

Based on the palaeomagnetic results from Lake Biwa, Japan, Yaskawa (1974) discussed the simple model about where the magnetic moment is fixed. Otofuji and Sasajima (1981) made the systematic 
experiments of the gravitational compaction and showed that the post-depositional DRM has the proportionality to the external field and the additivity during compaction.

$$
\begin{gathered}
J\left(\rho_{1}, \rho_{2}, \boldsymbol{H}_{\mathrm{ex}}\right) \propto \boldsymbol{H}_{\mathrm{ex}}, \\
J\left(\rho_{0}, \rho_{n}, \boldsymbol{H}_{\mathrm{ex}}\right)=\sum_{i=1}^{n} \boldsymbol{J}\left(\rho_{i-1}, \rho_{i}, \boldsymbol{H}_{\mathrm{ex}}\right),
\end{gathered}
$$

where $\rho_{i}$ is the density of sediment at the $i$-th step of compaction, and $J\left(\rho_{i}, \rho_{j}, \boldsymbol{H}_{\mathrm{ex}}\right)$ is the post-depositional DRM acquired between the $i$-th and $j$-th steps in an external field $\boldsymbol{H}_{\text {ex }}$. Combining these relations, Otofuji and Sasajima (1981) proposed the following formula of the post-depositional DRM mechanism.

$$
\boldsymbol{J}(t) \propto \int_{0}^{t} \boldsymbol{H}_{\mathrm{ex}}(t-\tau) F(\rho(\tau)) \frac{\partial \rho(\tau)}{\partial \tau} d \tau,
$$

where $F(\rho)$ is a characteristic function of the density change.

Payne and Verosub (1982) experimentally estimated the amount of remagnetized fraction in the dewatered sediment. Rotating the sample by $90^{\circ}$ in a constant field of a zero declination during the dewatering process, the change in the direction of the resultant magnetization was measured. If all the magnetic particles have the same magnetic moment, the declination $(D)$ is a function of remagnetized fraction $(g)$ as below (Payne and Verosub, 1982).

$$
D=\tan ^{-1}\{g / 1-g\}
$$

They also reported that remagnetization was limited to the sandy samples.

Hyodo (1984) explicitly explained the post-depositional DRM mechanism by the convolution of the geomagnetic field. He proposed a generalized formula,

$$
J(t) \propto \int_{0}^{t} \boldsymbol{H}_{\mathrm{ex}}(t-\tau) r(\tau) d \tau,
$$

where $r(t)$ is a response function. This response function is called a fixing function of magnetic moments and is regarded as the term of $\{F(\rho) \partial \rho / \partial \tau\}$ in Eq. (3). Equation (5) is applicable to any linear system if the response function is known. Hyodo (1984) approximated the response function during the compaction with an exponential one,

$$
r(\tau)= \begin{cases}A \exp (-A \tau) & (\tau \geq 0) \\ 0 & (\tau<0)\end{cases}
$$

where $\int r(\tau) d \tau=1$, and $A$ is the fixing factor related to the fixing depth. This factor is equivalent to the halffixing depth $(Z)$ at which half of magnetic moments are locked. The relationship between the half-fixing depth and the sedimentation rate $(z)$ is deduced to be

$$
A=(z / Z) \ln 2 .
$$

Hyodo (1984) also proposed the method for the deconvolution of the post-depositional DRM as 
follows. The post-depositional DRM directions observed from the homogeneous sediment are defined as $\boldsymbol{m}(t)$.

$$
\boldsymbol{m}(t)=\int_{0}^{t} f(\tau) r(t-\tau) d \tau /\left|\int_{0}^{t} \boldsymbol{f}(\tau) r(t-\tau) d \tau\right|
$$

If $\left|\int f(\tau) r(t-\tau) d \tau\right|$ is constant and taken to be unity,

$$
\boldsymbol{m}(t)=\int_{0}^{t} \boldsymbol{f}(\tau) r(t-\tau) d \tau \approx \int_{-\infty}^{+\infty} \boldsymbol{f}(\tau) r(t-\tau) d \tau,
$$

where $t \gg A^{-1}$ is assumed. We tentatively discuss the one-dimensional case. Defining the Fourie transform of $m(t), f(t)$ and $r(t)$ as $M(\omega), F(\omega)$ and $R(\omega)$, respectively, Eq. (9) becomes

$$
M(\omega)=F(\omega) R(\omega)
$$

Hence the Fourie transform of the original field is deduced.

$$
F(\omega)=M(\omega) / R(\omega)
$$

Hyodo (1984) applied the finite Fourie transform to the data set of the observed directions, and deconvolved it into the original geomagnetic field. Because the response function is assumed as in Eq. (6), the phase is advanced and its amplitude is declined in the post-depositional DRM. This effect is severe for high frequency components, which results in smoothing the original field like a low-pass filter. Hyodo's method is a conventional technique taking account of the post-depositional DRM mechanism. However, several important problems are remaining in his method as follows.

1) The validity of the exponential-type response function is unclear.

2) The convolved field intensity, $\left|\int f(\tau) r(t-\tau) d \tau\right|$, is assumed to be constant. This assumption reduces three-dimensional deconvolution into two-dimensional one of the inclination and declination. However, this assumption sometimes becomes unreasonable. For example, the rapid variation in the geomagnetic field direction possibly causes the intensity change in the post-depositional DRM as observed in the polarity transition. Directions of the post-depositional DRM are basically coupled with both the intensity and the direction of the geomagnetic field.

3) The plausible fixing factor is assumed before the deconvolution. Otherwise, it must be estimated by fitting the deconvolution result to the already known geomagnetic field.

4) The deconvolution is performed in the frequency domain using the finite Fourie transform. This procedure is valid for approximating the original data. However, the deconvolution by Eq. (11) assumes that the geomagnetic field really changes with the finite and discrete frequencies. These frequencies obviously depend upon the time span covered by the analysed data.

5) After the deconvolution, high frequency components are growing. In Hyodo's method, no criterion is given for how to distinguish signals from noises in the high frequency region.

In order to avoid these difficulties, a new deconvolution method is proposed in the next section.

\section{New Formulation of the Post-Depositional DRM Deconvolution}

It is possible that the post-depositional DRM direction is measured for samples of the same stratigraphic horizons from different sites. The present study assumes four following conditions. 1) The sites are located close to each other and then the external field is regarded as the same. 2) The measurement is performed for the continuous columnar section from each site. 3) The post-depositional DRM direction 
is observed to be somewhat different. 4) The discrepancy of the measured directions arises mainly from the difference of response functions. For example, when the fixing depth is the same for all the sites, the response function is modulated in the time domain if the sedimentation rate is different between sites.

Convolving the external fields depends upon not only field directions but field intensities. As a consequence the deconvolution of post-depositional DRM directions results in estimating three components of original fields and response functions from measured (that is, convolved) directions. If a certain constraint is assumed in the analysis, the deconvolution can be done concerning with only the field directions like Hyodo's deconvolution.

\subsection{Response function}

As mentioned before, the observations and the laboratory experiments of natural and synthetic sediments imply that the density, water content and void ratio of sediments abruptly change after the deposition. The intensive bioturbation, which is considered to cause remagnetization of sediments (e.g. Verosub, 1977), often occurs within some depth below the depositional surface. These changes may be phenomenologically approximated with an exponential function because the degree of compaction or biogenic activity is regarded to be gradually declined with depth. However the detailed features are still unknown. In this section, a possible physical basis will be given for applying an exponential fixing function.

The gravitational compaction, which is considered to generate the post-depositional DRM (e.g. Hamano, 1980), is theoretically examined in a simple case. Suppose the sediment column with no lateral movement and the downward stress at the surface. The water in the sediment is assumed to drain only through the sediment surface. The displacement at the surface gradually increases with time and finally converges to the following value according to the equation by Jaeger and Cook (1979).

$$
\Delta h=-\Delta \sigma h /(\lambda+2 G)
$$

where $h$ is length of the sediment column, $\Delta h$ is an extensional displacement, $\Delta \sigma$ is a downward stress, and $\lambda$ and $G$ are Lame's parameters. This relationship resembles that for the elastic body but the Lame's parameters depend upon the stress in the sediment. The differential equation (12) can be solved as follows.

$$
h=h_{0} \exp \left\{-\int_{0}^{\sigma} d \sigma /(\lambda+2 G)\right\}
$$

where $h_{0}$ is an initial value of $h$.

The displacement of the surface is equivalent to the amount of the water discharged through a unit surface. Hence the change in water content $(\Delta w)$ becomes

$$
\Delta w \propto h-h_{0}=h_{0}\left[\exp \left\{-\int_{0}^{\sigma} d \sigma /(\lambda+2 G)\right\}-1\right] .
$$

In the deposited sediment, the stress corresponds to the effective pressure exerted by the overburden sediment. The pressure increases as the sedimentation progresses.

Payne and Verosub (1982) suggested that the sample showing the almost complete remagnetization has an approximately linear relationship between the measured declination and the water content. Further formulation is attempted in the present study for the link to compaction. The observed declination $(D)$ in their experiment is roughly approximated with the following function of the water content $(w)$.

$$
D=\pi w /\left(2 w_{0}\right)
$$


where $w_{0}$ is an initial water content. From Eqs. (4) and (15),

$$
\begin{aligned}
g & =\tan \left\{\pi w /\left(2 w_{0}\right)\right\} /\left[1+\tan \left\{\pi w /\left(2 w_{0}\right)\right\}\right] \\
& \approx w / w_{0} .
\end{aligned}
$$

This approximation is valid except for the vicinity of $w / w_{0} \sim 0$. A magnitude of $g$ is equivalent to the intensity fraction of the post-depositional DRM acquired during the dewatering process as the water content changes from $w$ to zero. Because the water content in sediments is a function of time $(\tau)$ since deposition, a post-depositional DRM $J(t)$ is expressed as

$$
J(t) \propto \int_{0}^{t} \boldsymbol{H}_{\mathrm{ex}}(t-\tau) \frac{\partial g}{\partial \tau} d \tau=\int_{0}^{t} \boldsymbol{H}_{\mathrm{ex}}(t-\tau) \frac{\partial w(\tau)}{w_{0}(t) \partial \tau} d \tau,
$$

where $\boldsymbol{H}_{\mathrm{ex}}$ is the external magnetic field and $t$ is taken to be the time before the present.

From Eqs. (5) and (14), the response function corresponds to $\partial w / \partial t$ and therefore,

$$
r(t) \propto\left\{h_{0} /(\lambda+2 G)\right\}(\partial \sigma / \partial t) \exp \left\{-\int_{0}^{\sigma} d \sigma /(\lambda+2 G)\right\}
$$

If the density contrast between the sediment and the water is constant $(=\Delta \rho)$ and the sedimentation rate is also constant $(=z)$,

$$
\sigma(t)=z t \Delta \rho
$$

In the assumption of the constant Lame's parameters,

$$
\begin{aligned}
& r(t)=\beta \exp (-\beta t), \\
& \beta=z \Delta \rho /(\lambda+2 G)
\end{aligned}
$$

where the normalization factor is considered. Therefore, the gravitational compaction in the very simplified case yields the exponential-type response function.

These results from the observation and the theory suggest that the exponential response function is a reasonable first-order approximation, though the real response function is predicted to be more complicated than a simple exponential function.

\subsection{Basic equations}

As the features of the geomagnetic field are basically unknown, the present deconvolution method adopts no specific functions for describing the original field.

The post-depositional DRM directions of continuous columnar sections from $N$ sites are assumed to have been measured as the time series against $t_{j}(j=1, \ldots, n)$ with an equal time interval $\Delta t$. Those unit vectors are defined as $\left\{\boldsymbol{m}_{i, j}\left(m_{x i j}, m_{y i j}, m_{2 i j}\right) \mid i=1, \ldots, N ; j=1, \ldots, n\right\}$. The response function for the $i$-th site is assumed to be

$$
r_{i}(t)= \begin{cases}A_{i} \exp \left(-A_{i} t\right) & (t \geq 0) \\ 0 & (t<0)\end{cases}
$$


where the response function is normalized and $A_{i}$ is a fixing factor of the $i$-th site. $A_{i}$ is related to the sedimentation rate $\left(z_{i}\right)$ and the half-fixing depth $\left(Z_{i}\right)$ as in Eq. (7).

$$
A_{i}=\left(z_{i} / Z_{i}\right) \ln 2
$$

The geomagnetic field is convolved with the response function to result in a vector $\boldsymbol{M}_{i, j}$.

$$
\begin{aligned}
& \boldsymbol{M}_{i, j}=\int_{0}^{t_{j}} f(\tau) A_{i} \exp \left\{-A_{i}\left(t_{j}-\tau\right)\right\} d \tau, \\
& M_{i, j}=\left|\boldsymbol{M}_{i, j}\right|,
\end{aligned}
$$

where $\boldsymbol{f}(\tau)$ is the geomagnetic field vector at $t=\tau$. If there is no noise, $\boldsymbol{m}_{i, j}$ is parallel to $\boldsymbol{M}_{i, j}$.

$$
\boldsymbol{m}_{i, j}=\boldsymbol{M}_{i, j} / M_{i, j}
$$

This becomes the following equations like a recursive formula (see Appendix A).

$$
\overline{\boldsymbol{f}}_{j}=c_{i}^{-} M_{i, j} \boldsymbol{m}_{i, j}+c_{i}^{+} M_{i, j+1} \boldsymbol{m}_{i, j+1}
$$

where

$$
\begin{aligned}
& \overline{\boldsymbol{f}}_{j}=\frac{1}{\Delta t} \int_{t_{j}}^{t_{j+1}} \boldsymbol{f}(t) d t, \\
& c_{i}^{ \pm}=\frac{1}{2} \pm \frac{1}{A_{i} \Delta t} .
\end{aligned}
$$

These equations mean that an averaged field in the $j$-th division is given by the weighted combination of the post-depositional DRM directions at two endpoints.

If $M_{i, j}=M_{i, j+1}$, Eq. (27) is rewritten to be

$$
\boldsymbol{f}_{i, j}^{*}=\overline{\boldsymbol{f}}_{j} / M_{i, j}=c_{i}^{-} \boldsymbol{m}_{i, j}+c_{i}^{+} \boldsymbol{m}_{i, j+1} \quad(j=1, \ldots, n-1)
$$

where $f_{i, j} *$ represents the field direction averaged for the $j$-th time interval. This makes it possible to estimate the original field direction from the post-depositional DRM direction. This deconvolution is very simple and useful, but the problem is whether or not the condition of $M_{i, j}=M_{i, j+1}$ is satisfied. Applying the expression of $M_{i, j+1}=M_{i, j}+\Delta M_{i, j}$ and $m_{i, j+1}=m_{i, j}+\Delta m_{i, j}$ to Eq. (27),

$$
\overline{\boldsymbol{f}}_{j}=M_{i, j} \boldsymbol{m}_{i, j}+c_{i}^{+}\left(M_{i, j} \Delta \boldsymbol{m}_{i, j}+\Delta M_{i, j} \boldsymbol{m}_{i, j}+\Delta M_{i, j} \Delta \boldsymbol{m}_{i, j}\right)
$$

The cross term, $\Delta M_{i, j} \Delta m_{i, j}$, is negligible when $\Delta M_{i, j}$ and $\Delta m_{i, j}$ are small. The term of $M_{i, j} \Delta m_{i, j}$ is a component perpendicular to $\boldsymbol{M}_{i, j}$ while the term of $\Delta M_{i, j} \boldsymbol{m}_{i, j}$ is a parallel component. If the parallel component is much smaller than the perpendicular component, the above equation is equivalent to Eq. (30). This condition means that the convolved field varies its intensity much more gradually than its direction. 
Equation (30) is modified to the following form.

$$
\boldsymbol{f}_{i, j}^{*}=\left(1+c_{i}^{+} \Delta M_{i, j} / M_{i, j}\right) \boldsymbol{m}_{i, j}+c_{i}^{+} \Delta \boldsymbol{m}_{i, j+1},
$$

where the higher order term is neglected. From Eq. (32), Eq. (30) is applicable in the condition of $c_{i}{ }^{+} \Delta M_{i, j} / M_{i, j} \ll<1$. As $\Delta \boldsymbol{m}_{i, j}$ is perpendicular to $\boldsymbol{m}_{i, j}$, the directional variation in $\boldsymbol{f}_{i, j}{ }^{*}$ is sensitive to $\Delta \boldsymbol{m}_{i, j}$ rather than $\Delta M_{i, j}$. Besides, $\Delta \boldsymbol{m}_{i, j}$ is observable but $\Delta M_{i, j}$ is not. This situation suggests that the present deconvolution is more valid for the estimation of the directional change than of the intensity change. An example of this validity will be shown later.

\subsection{Practical formulation}

The present method assumes only the continuous change in the geomagnetic field in order to restore the original field. This requires that the time sequence of convolved fields smoothly changes and gives a statistical way to distinguish signals from noises.

3.3.1. Neglecting the intensity change in the convolved field

When the fixing factor is known and the intensity of the convolved field nearly satisfies the condition of $M_{i, j}=M_{i, j+1}$, the post-depositional DRM directions are immediately deconvolved by applying Eq. (30). This deconvolution technique is extremely simpler than Hyodo's method using the finite Fourie transform.

The fixing factor is unknown in most cases. When the approximation of $M_{i, j}=M_{i, j+1}$ is allowed, there may be a few ways to estimate the plausible fixing factor and to deconvolve the post-depositional DRM directions, as follows.

(a) Compared with the established master curve such as archaeomagnetic directions, we can estimate the fixing factor and apply it to the older time span of the same site.

(b) If continuous sections from multiple sites recorded the same geomagnetic field with different fixing factors, some deconvolution technique gives the original field together with the fixing factor.

In the case of (a), the following least square fitting of the direction can be applied because the length of $f_{i, j} *$ is almost unity.

$$
S=\sum_{j=1}^{n-1}\left\{\boldsymbol{f}_{i, j}^{*}-\boldsymbol{f}_{d}\left(t_{j}\right)\right\}^{2},
$$

where $f_{d}\left(t_{j}\right)$ is the palaeodirection vector according to the master curves. Minimizing $S$ gives the optimal value of the fixing factor for the $i$-th sedimentary section. This problem is linear about $A_{i}^{-1}$ and the equations are obtained in the condition of $\partial S / \partial A_{i}{ }^{-1}=0$. The direction of the original field is calculated from Eq. (30) by the deconvolution adopting the obtained value of $A_{i}$. The intensity change cannot be estimated in this case. Hyodo et al. (1993) applied the FFT deconvolution method to the marine and lake sediments from Japan in comparison with the archaeomagnetic curves. In their analysis the sum of inner products, $\boldsymbol{f}^{*} \cdot \boldsymbol{f}_{\boldsymbol{d}}$, is maximized through the iteration of the nonlinear problem.

In the case of (b), $S$ is defined as follows.

$$
S=\sum_{k=1}^{N} \sum_{1>k}^{N} \sum_{j=1}^{n-1}\left\{\boldsymbol{f}_{k, j}^{*}-\boldsymbol{f}_{i, j}^{*}\right\}^{2} .
$$

The minimization of $S$ is accomplished in the conditions of $\partial S / \partial A_{i}^{-1}=0(i=1, \ldots, N)$ similarly to the case of (a). 


\subsubsection{Considering the intensity change in the convolved field}

The constancy of the convolved field intensity $\left(M_{i, j}=M_{i, j+1}\right)$ is assumed in the above discussion. To generalize the problem, $M_{i, j}$ is treated as a variable. Analysing the post-depositional DRM directions from multiple sites, the relative intensity of the original field is estimated as well as the original field direction and the fixing factor.

In that case, the basic relationship between the $k$-th and $l$-th sites is given as follows.

$$
\begin{array}{r}
\left(c_{k}^{-} M_{k, j} \boldsymbol{m}_{k, j}+c_{k}^{+} M_{k, j+1} \boldsymbol{m}_{k, j+1}\right)-\left(c_{l}^{-} M_{l, j} \boldsymbol{m}_{l, j}+c_{l}^{+} M_{l, j+1} \boldsymbol{m}_{l, j+1}\right) \\
=\boldsymbol{O}(0,0,0)+\varepsilon_{k l, j} \quad(j=1, \ldots, n-1)
\end{array}
$$

where $\varepsilon_{k l, j}$ is a random-noise vector and $\boldsymbol{O}(0,0,0)$ is a zero vector. Each component of $\varepsilon_{k l, j}$ is assumed to be independently subjected to a normal distribution $N\left(0, \sigma^{2}\right) . \Lambda=\left(M_{1,1}, \ldots, M_{1, n}, \ldots, M_{N, 1} \ldots, M_{N, n}\right)^{\mathrm{T}}$ and $\boldsymbol{A}$ $=\left(A_{1}, A_{2}, \ldots, A_{N}\right)^{\mathrm{T}}$ are taken as unknown parameters. The following condition is assumed because $M_{k, j}$ is a relative intensity of the convolved field.

$$
|\mathbf{\Lambda}|=1
$$

The coincidence level of the deconvolved fields may be evaluated with $S$.

$$
S=\sum_{k=1}^{N-1} \sum_{1=k+1}^{N} \sum_{j=1}^{n-1}\left|\boldsymbol{g}_{k j}-\boldsymbol{g}_{l j}\right|^{2},
$$

where

$$
\boldsymbol{g}_{k j}=c_{k}^{-} M_{k, j} \boldsymbol{m}_{k, j}+c_{k}^{+} M_{k, j+1} \boldsymbol{m}_{k, j+1} \text {. }
$$

$S$ is expressed in a quadratic form using a matrix $V$ (see Appendix B).

$$
S=\boldsymbol{\Lambda}^{\mathrm{T}} V^{\mathrm{T}} V \boldsymbol{\Lambda}
$$

The evaluation with $S$ assumes a constant noise level, $\sigma^{2}$, despite of the various length of the deconvolved field vector $\boldsymbol{g}_{k j}$. Although this assumption may cause a relatively low resolution in the weak intensity part, the least square fitting by minimization of $S$ makes it possible to obtain the solution of $\Lambda$ as a linear problem. The magnitude of $S$ tends to be large as the assumed fixing factors decrease. This is because the length of a deconvolved vector $g_{k j}$ is depending upon $A_{k}$ as in Eqs. (29) and (38). Therefore some improvement such as normalization is necessary for the comparison of $S$ for different fixing factors.

The angular dispersion between the deconvolved field vectors may represent the goodness-of-fit, using the Fisher's precision parameter $\kappa$. Simplifying the problem, we discuss the case of two sites $(N=$ 2 ). In that case, the averaged value of the precision parameter $\left(\kappa_{0}\right)$ is expressed by those of the restored vectors for individual divisions $\left(\boldsymbol{\kappa}_{j}\right)$.

$$
1 / \kappa_{0}=\left(\sum_{j=1}^{n-1} 1 / \kappa_{j}\right) /(n-1) .
$$

The normalized variance $\sigma_{0}{ }^{2}$, corresponding to $\kappa_{0}$, can be written as 


$$
\sigma_{0}^{2}=2 / \kappa_{0}
$$

Defining the averaged vector of the deconvolved fields as

$$
\boldsymbol{F}_{j}=\left(\boldsymbol{g}_{1 j}+\boldsymbol{g}_{2 j}\right) / 2
$$

then, after Tsunakawa (1992), the individual precision parameters become

$$
\begin{aligned}
& \kappa_{j}=2 F_{j}^{2} / \sigma^{2}, \\
& \sigma^{2}=S /(n-1),
\end{aligned}
$$

where $F_{j}=\left|\boldsymbol{F}_{j}\right|$. Consequently, the relationship between the normalized variance and the ordinary variance is deduced from Eqs. (40), (41) and (43).

$$
\sigma_{0}^{2}=\sigma^{2} \Sigma_{F}
$$

where

$$
\Sigma_{F}=\frac{1}{n-1} \sum_{j=1}^{n-1} F_{j}^{-2} .
$$

It is recognized in Eq. (45) that the dependency of $\sigma^{2}$ upon $F_{j}^{2}$ is canceled by $\Sigma_{F}$.

Considering each component of $\varepsilon_{12 j}$, the likelihood becomes

$$
L(\boldsymbol{\Lambda}, \boldsymbol{A}) \propto\left(3 / \sigma_{0}^{2}\right)^{3(n-1) / 2}=\left(\sigma^{2} \Sigma_{F} / 3\right)^{-3(n-1) / 2} .
$$

Therefore the optimal solution is obtained by minimizing the logarithmic likelihood, $\Theta$.

$$
\Theta=-2 \ln L=3(n-1) \ln \left(\sigma^{2} \Sigma_{F}\right)+\text { const. }
$$

In practice, assuming the fixing factors, the eigenvalue and eigenvector of $V^{\mathrm{T}} V$ are calculated in the condition of $|\Lambda|=1$. The minimum eigenvalue gives $\sigma^{2}$, and the corresponding eigenvector yields $\boldsymbol{F}_{j}$. Then $\Theta$ is calculated and compared with others for various fixing factors. This becomes a nonlinear problem of fixing factors.

The observed data generally contain noises due to experimental errors, instability of magnetization, inhomogeneity of sediments and so on. Applying the smoothing method to the direction data, the noise may efficiently be removed. If many data sets are available, the reliable solution may be obtained by minimizing $\Theta$. However, as only two or three data sets are usually analysed, the effect of the remaining noises becomes considerable. We need a more sophisticated procedure in the deconvolution in practice.

The original field is supposed to continuously change as stated earlier. This assumption gives a reasonable constraint that an intensity of a convolved field smoothly changes. This constraint is taken into account as the Bayesian procedure for the noise reduction.

From Eqs. (35), (39) and (44), the likelihood function of $\Lambda$ and $\sigma^{2}$ is written as below. 


$$
L\left(\boldsymbol{\Lambda}, \sigma^{2} \mid \boldsymbol{A}\right)=\frac{1}{\left(2 \pi \sigma^{2} / 3\right)^{3(n-1) / 2}} \exp \left\{-\frac{S}{2\left(\sigma^{2} / 3\right)}\right\}
$$

The smooth change in the convolved field intensity requires small values of the second difference of $\left\{M_{k j}\right\}$. According to the Bayesian procedure by Akaike (1980), the difference is assumed to be subjected to a normal distribution $N\left(0, \sigma_{k}^{2}\right)$. Using a hyperparameter $u_{k}^{2}\left(=\sigma^{2} / 3 \sigma_{k}^{2}\right)$, the prior distribution of $\Lambda$ is expressed as below.

$$
\pi\left(\boldsymbol{\Lambda}, \sigma^{2} \mid\left\{u_{1}^{2}, u_{2}^{2}\right\}, \boldsymbol{A}\right)=\left(\frac{u_{1}^{2 n} u_{2}^{2 n}}{\left(2 \pi \sigma^{2} / 3\right)^{2 n}}\right)^{1 / 2} \exp \left\{-\frac{S_{u}}{2\left(\sigma^{2} / 3\right)}\right\}
$$

where

$$
\begin{aligned}
& S_{u}=\sum_{k=1}^{2} u_{k}^{2} \sum_{j=1}^{n}\left|\Delta M_{k, j}\right|^{2}, \\
& \Delta M_{k, j}=M_{k, j}-2 M_{k, j-1}+M_{k, j-2} .
\end{aligned}
$$

The second difference of the convolved field corresponds to the first derivative of the original field because of Eq. (24). For the mathematical characteristics, three components of the original field must be continuous and first differentiable. This is natural for the geomagnetic field.

According to the Bayes theorem, a likelihood function $L(x \mid y)$, a prior distribution $\pi(x)$, and a probability $p(y)$ provide a posterior distribution $\pi_{\text {post }}(x \mid y)$, where $\boldsymbol{y}$ is a data set and $\boldsymbol{x}$ is an unknown parameter set.

$$
\pi_{\text {post }}(\boldsymbol{x} \mid \boldsymbol{y}) \propto L(\boldsymbol{x} \mid \boldsymbol{y}) \pi(\boldsymbol{x}) / p(\boldsymbol{y}) .
$$

In the ordinary Bayesian procedure, the data $y$ is the observation independent of the parameter $x$. Therefore $p(y)$ is constant and the next relation is adaptable to the estimation of $x$.

$$
\pi_{\text {post }}(\boldsymbol{x} \mid \boldsymbol{y}) \propto L(\boldsymbol{x} \mid \boldsymbol{y}) \pi(\boldsymbol{x})
$$

In the present analysis, however, a data set $\boldsymbol{y}$ becomes $\boldsymbol{O}(0,0,0)$ in Eq. (35), namely $\boldsymbol{g}_{1 j}=\boldsymbol{g}_{2 j}$, which is not observable but theoretical. As discussed before, $p\left(\boldsymbol{y}=\boldsymbol{O} ; \boldsymbol{g}_{1 j}=\boldsymbol{g}_{2 j} \mid\left\{u_{1}{ }^{2}, u_{2}{ }^{2}\right\}, \boldsymbol{A}\right)$ depends upon the length of the deconvolved field vector $F_{j}$ which is a function of $A$ and $\left\{u_{1}{ }^{2}, u_{2}{ }^{2}\right\}$. For example, $p\left(O \mid\left\{u_{1}{ }^{2}\right.\right.$, $\left.u_{2}^{2}\right\}, A$ ) becomes small as to the increasing of $F_{j}$. Hence the present analysis explicitly take account of $p\left(\boldsymbol{O} \mid\left\{u_{1}^{2}, u_{2}^{2}\right\}, \boldsymbol{A}\right)$. The probability of $p\left(O \mid\left\{u_{1}{ }^{2}, u_{2}^{2}\right\}, \boldsymbol{A}\right)$ can be evaluated by the factor $\Sigma_{F}$ in Eq. (46) and thus,

$$
p\left(\boldsymbol{O} \mid\left\{u_{1}^{2}, u_{2}^{2}\right\}, A\right) \propto\left(\Sigma_{F}\right)^{3(n-1) / 2} .
$$

In this context, the likelihood function is expressed as $L\left(\Lambda, \sigma^{2} \mid \boldsymbol{O},\left\{u_{1}^{2}, u_{2}^{2}\right\}, \boldsymbol{A}\right)$. Hence the posterior 
distribution $\pi_{\text {post }}$ of $\Lambda$ and $\sigma^{2}$ becomes

$$
\begin{aligned}
& \pi_{\text {post }}\left(\boldsymbol{\Lambda}, \sigma^{2} \mid \boldsymbol{O},\left\{u_{1}^{2}, u_{2}^{2}\right\}, \boldsymbol{A}\right) \\
& \propto L\left(\boldsymbol{\Lambda}, \sigma^{2} \mid \boldsymbol{O},\left\{u_{1}^{2}, u_{2}^{2}\right\}, \boldsymbol{A}\right) \pi\left(\boldsymbol{\Lambda}, \sigma^{2} \mid\left\{u_{1}^{2}, u_{2}^{2}\right\}, \boldsymbol{A}\right) / p\left(\boldsymbol{O} \mid\left\{u_{1}^{2}, u_{2}^{2}\right\}, A\right) \\
& \propto\left(\frac{1}{\left(2 \pi \sigma^{2} / 3\right)^{3(n-1)}}\right)^{1 / 2}\left(\frac{u_{1}^{2 n} u_{2}^{2 n}}{\left(2 \pi \sigma^{2} / 3\right)^{2 n}}\right)^{1 / 2} \Sigma_{F}^{-3(n-1) / 2} \exp \left\{-\frac{S_{b}}{2\left(\sigma^{2} / 3\right)}\right\},
\end{aligned}
$$

where $S_{b}=S+S_{u}$. Log marginal likelihood of $\left\{u_{1}{ }^{2}, u_{2}{ }^{2}\right\}$ and $A$ is taken as a Bayesian information criterion (ABIC) proposed by Akaike (1980).

$$
\begin{aligned}
& \operatorname{ABIC}\left(\sigma^{2},\left\{u_{1}^{2}, u_{2}^{2}\right\}, \boldsymbol{A}\right) \\
& =-2 \ln \int_{\mathbf{\Lambda} \mid=1} \pi_{\text {post }}\left(\boldsymbol{\Lambda}, \sigma^{2} \mid\left\{u_{k}^{2}\right\}, \boldsymbol{A}\right) d \mathbf{\Lambda} \\
& =-2 \ln \int_{|\boldsymbol{\Lambda}|=1} L\left(\boldsymbol{\Lambda}, \sigma^{2}\right) \pi\left(\mathbf{\Lambda} \mid\left\{u_{k}^{2}\right\}, \boldsymbol{A}\right) \Sigma_{F}^{-3(n-1) / 2} d \mathbf{\Lambda}+\text { const. } \\
& =\{3(n-1)+2 n\} \ln \sigma^{2}-n\left(\ln u_{1}^{2}+\ln u_{2}^{2}\right)-2 \ln \int_{|\boldsymbol{\Lambda}|=1} \Sigma_{F}^{-3(n-1) / 2} \exp \left[-3 S_{b} / 2 \sigma^{2}\right] d \boldsymbol{\Lambda}+\text { const. }
\end{aligned}
$$

The sum $S_{b}$ can be expressed in a quadratic form by using a specific matrix $W$ (see Appendix C).

$$
S_{b}=\Lambda^{\mathrm{T}} W^{\mathrm{T}} W \boldsymbol{\Lambda}
$$

Minimizing $S_{b}$ in the condition of $|\Lambda|=1$ becomes an eigenvalue problem. Eigenvalues of $W^{\mathrm{T}} W$ are defined as $\left\{\mu_{i} ; i=1, \ldots, 2 n\right\}$ in the ascent order and their corresponding eigenvectors as $\left\{\Lambda_{i} ; i=1, \ldots, 2 n\right\}$. Because $W^{\mathrm{T}} W$ is a positive-definite symmetric matrix, all of $\mu_{i}$ are positive and $\mu_{1}$ gives the minimum of $S_{b}$. D)

Transforming and approximating the integration term in Eq. (57), ABIC is given to be (see Appendix

$$
\begin{aligned}
& \operatorname{ABIC}\left(\sigma^{2},\left\{u_{1}^{2}, u_{2}^{2}\right\}, \boldsymbol{A}\right) \\
& =\{3(n-1)+2 n\} \ln \sigma^{2}-n\left(\ln u_{1}^{2}+\ln u_{2}^{2}\right) \\
& +3(n-1) \ln \Sigma_{F}^{*}+3 \mu_{1} / \sigma^{2}+\sum_{i=2}^{2 n} \ln \left\{\left(\mu_{i}-\mu_{1}\right) / \sigma^{2}\right\}+\text { const. }
\end{aligned}
$$

where $\Sigma_{F} *$ is calculated for $\Lambda_{1}$ from Eqs. (42) and (46). Considering $\partial \mathrm{ABIC} / \partial \sigma^{2}=0$, the estimate of $\sigma^{2}$ becomes $\sigma^{* 2}$, which is

$$
\sigma^{* 2}=3 \mu_{1} /\{3(n-1)+1\} .
$$


Therefore,

$$
\begin{aligned}
& \operatorname{ABIC}\left(\left\{u_{1}^{2}, u_{2}^{2}\right\}, \boldsymbol{A}\right) \\
& =\{3(n-1)+1\} \ln \mu_{1}+\sum_{i=2}^{2 n} \ln \left(\mu_{i}-\mu_{1}\right)-\left(n \ln u_{1}^{2}+\ln u_{2}^{2}\right)+3(n-1) \ln \Sigma_{F}^{*}+\text { const. }
\end{aligned}
$$

Generalizing the above equation to data sets from $N$ sites,

$$
\begin{aligned}
& \operatorname{ABIC}\left(\left\{u_{k}^{2}: k=1, \ldots, N\right\}, A\right) \\
= & \{3 N(N-1)(n-1) / 2+1\} \ln \mu_{1}+\sum_{i=2}^{N n} \ln \left(\mu_{i}-\mu_{1}\right) \\
& -n \sum_{k=1}^{N} \ln u_{k}^{2}+\{3 N(N-1)(n-1) / 2\} \ln \Sigma_{F}^{*}+\text { const. }
\end{aligned}
$$

The optimal solutions of $\boldsymbol{A}$ and $\left\{u_{k}^{2}\right\}$ are obtained by minimizing the above ABIC as a nonlinear problem. Calculating ABIC at grid points of $\boldsymbol{A}$ and $\left\{u_{k}^{2}\right\}$ is practically useful for roughly searching a convergence point. Equation (29) requires $A_{k}<2$. If $A_{k}=2$, the post-depositional DRM directions are the same as those of the original fields because of no convolution. The term $\left\{u_{k}^{2}\right\}$ is expressed by an integer $P$ in the form of $u_{k}^{2}=2^{P}$, where usually $P=-5 \sim 10$ (Akaike, 1980). If there seems to be a local minimum of ABIC, one gives temporary initial values of $\boldsymbol{A}$ and $\left\{u_{k}^{2}\right\}$ and then solves the nonlinear problem with a certain method, for example, the DFP method (Fletcher and Powell, 1963). If a local minimum is not found, the searched area will be extended. All elements of $\Lambda_{1}$ should be positive. If no local minimum exists or some elements of $\Lambda_{1}$ are negative, it is suggested that the data sets are too noisy or inconsistent with the assumptions of the present deconvolution method. In other words, the present method is thought to be possessed of checking the consistency between the data sets and between the data and the assumptions in the deconvolution.

After obtaining the optimal solutions of $\boldsymbol{A}$ and $\boldsymbol{\Lambda}$, the original field is calculated from Eq. (36). This estimation is carried out for each section and the averaged field $\boldsymbol{g}_{j}{ }^{*}$ is taken as the optimal field vector.

$$
\boldsymbol{g}_{j}^{*}=\left(\sum_{k=1}^{N} \boldsymbol{g}_{k j}^{*}\right) / N
$$

The confidence limit is represented by $\sigma^{*}$. The $95 \%$ confidence levels of the total intensity, the inclination and the declination are calculated as in Appendix E.

If the sedimentary material is homogeneous between the sampling sites, the fixing depth is probably similar to each other. From Eq. (23), the fixing factor of the homogeneous sediment depends mainly upon sedimentation rates.

$$
A_{k} / A_{1} \approx z_{k} / z_{1}
$$

For the gravitational compaction, this is theoretically deduced from Eq. (21) if the Lame's parameters have similar values. In the condition of Eq. (64), only one of the fixing factors becomes an unknown parameter. 


\section{Examination of the Deconvolution Method Using Synthetic Data}

The deconvolution method proposed here is examined using the synthetic data. First the original field is assumed and convolved with an exponential response function. Two sets of the original field are prepared. The data set $G_{1}$ has a constant intensity. The other, $G_{2}$, significantly changes the intensity of the original field.

The $G_{1}$ set is analogous with the secular variation associated with fluctuations. The convolved field was calculated for the $G_{1}$ set at sixty-four points for two different fixing factors: $A_{11}=0.2$ (the convolved field set $G_{11}$ ) and $A_{12}=0.125$ (the convolved field set $G_{12}$ ), where $\Delta t=1$ (Fig. 1). Both of the data sets almost satisfy the constancy of the convolved field intensity because of $M_{i, j}=1.00 \pm 0.02$.

The $G_{2}$ set, assuming a polarity reversal from the normal to the reversed, was convolved using two fixing factors of $A_{21}=0.4$ (the convolved field set $G_{21}$ ) and of $A_{22}=0.8$ (the convolved field set $G_{22}$ ), where $\Delta t=1$ (Fig. 2). For the sets of $G_{21}$ and $G_{22}$, the resultant direction was calculated at twenty-one points. The $G_{21}$ and $G_{22}$ were added by directional random noises $\left(\alpha_{95}=15^{\circ}\right)$ to be called $G_{21}{ }^{\prime}$ and $G_{22} 2^{\prime}$ (Fig. 3).

\subsection{A single site with constant $M_{i, j}$ and a known fixing factor $\left(G_{11}\right)$}

The data set $G_{11}$, which contains no noise except for round errors, is analysed. It is recognized in Fig. 1 that phases of short-period components are shifted toward the past direction. This indicates that the direction change appears in the convolved field earlier than in the original field.

Given $A_{11}=0.2$, the deconvolution of $G_{11}$ is performed with the recursive method of Eq. (30) and with the FFT method of Eq. (11). The resultant directions from the recursive method agree with the original field extremely well (Fig. 4). The FFT method also can trace the trend of original fields but is partly deviated and fluctuated, especially near the ends. This instability arises from the assumption of periodic variations in the FFT method.
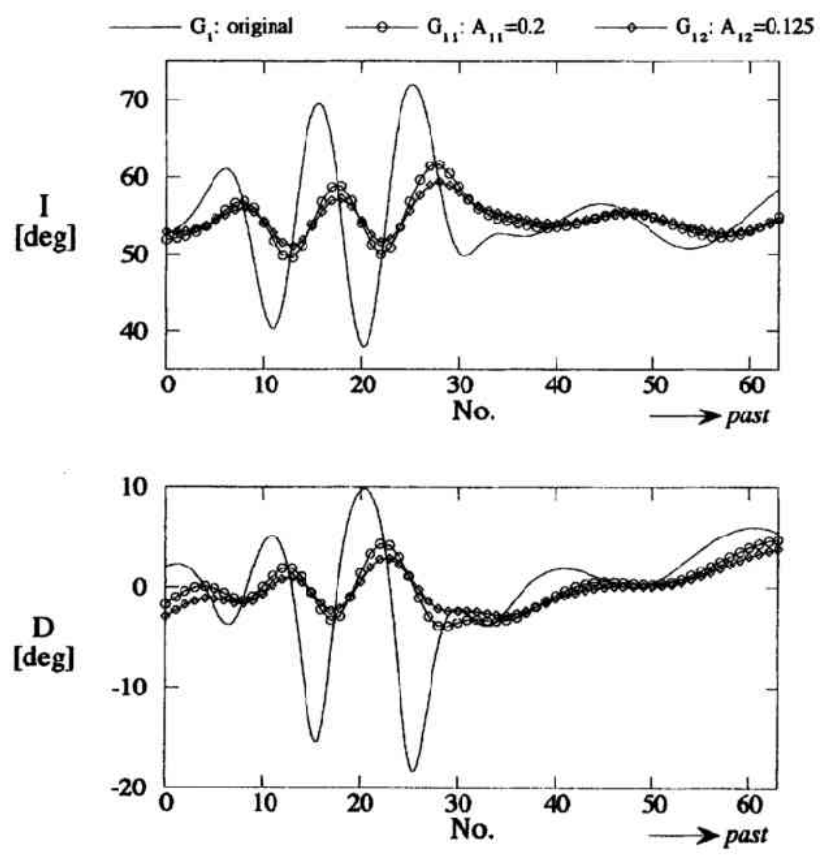

Fig. 1. Direction data sets used in the deconvolution assuming nearly constant intensities of the convolved field. $G_{1}$ : original, $G_{11}$ : convolved fields with a fixing factor of 0.2 , and $G_{12}$ : convolved fields with a fixing factor of 0.125 . 


\subsection{Two sites with constant $M_{i j}$ and different fixing factors $\left(G_{11}, G_{12}\right)$}

Short-period components show smaller amplitudes in the data set $G_{12}$ than in the $G_{11}$ (Fig. 1). This is because $A_{12}$ is smaller than $A_{11}$. Using Eq. (34), an optimal solution can be obtained with the least square method. The solution of fixing factors, $A_{11}=0.205$ and $A_{12}=0.128$, is concordant with the assumed values. The deconvolved directions agree well with the original field (Fig. 5).

\subsection{Two sites with variable $M_{i j}$ and different fixing factors $\left(G_{21}, G_{22}\right)$}

Comparing the patterns in Fig. 2, the change in $G_{21}$ occurs slightly earlier than in $G_{22}$. The postdepositional DRMs of $G_{21}$ are more influenced by the later normal field than those of $G_{22}$. These are caused by the condition of $A_{21}<A_{22}$.

As the data sets of $G_{21}$ and $G_{22}$ contain no noise, the optimal solution is obtained by minimizing $\Theta$ in Eq. (48). The contour map of $\Theta$ is shown in the coordinates of $A_{21}$ and $A_{22} / A_{21}$ (Fig. 6). The ratio between fixing factors is a useful parameter in the calculation because we often know which site has a smaller fixing factor. The upward concave feature is clearly recognized in this map and the convergence point is of
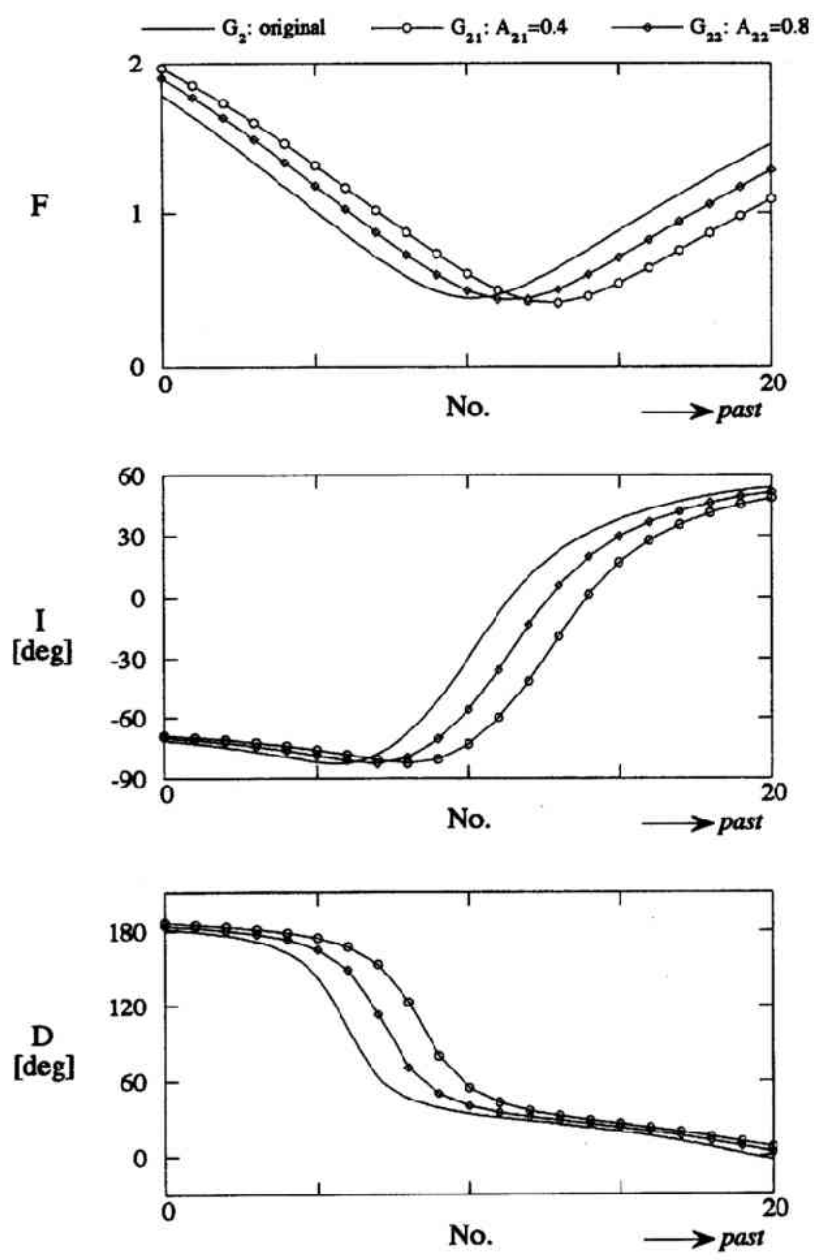

Fig. 2. Intensity and direction data sets for the deconvolution with variable intensities of the convolved field. $G_{2}$ : original, $G_{21}$ : convolved fields with a fixing factor of 0.4 , and $G_{22}$ : convolved fields with a fixing factor of 0.8 . The intensity data of $G_{21}$ and $G_{22}$ in the uppermost figure are not used in the analysis. 

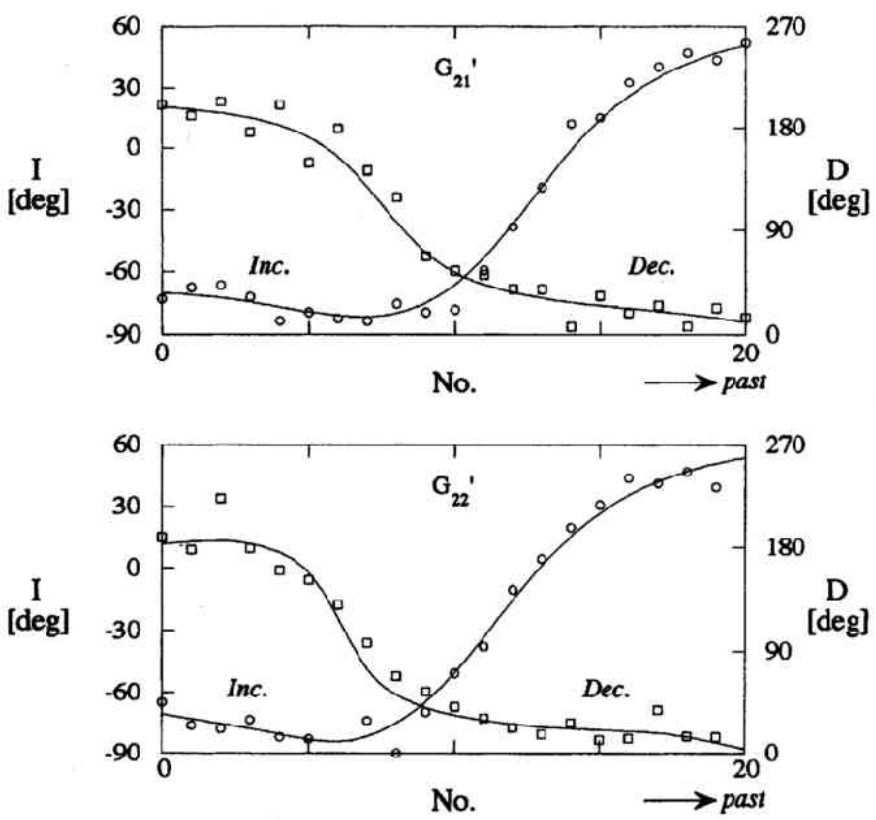

Fig. 3. Direction data sets added by random noises and the smoothed curves. Circles: inclinations, and squares: declinations. The upper: $G_{21}$ and the lower: $G_{22}$.
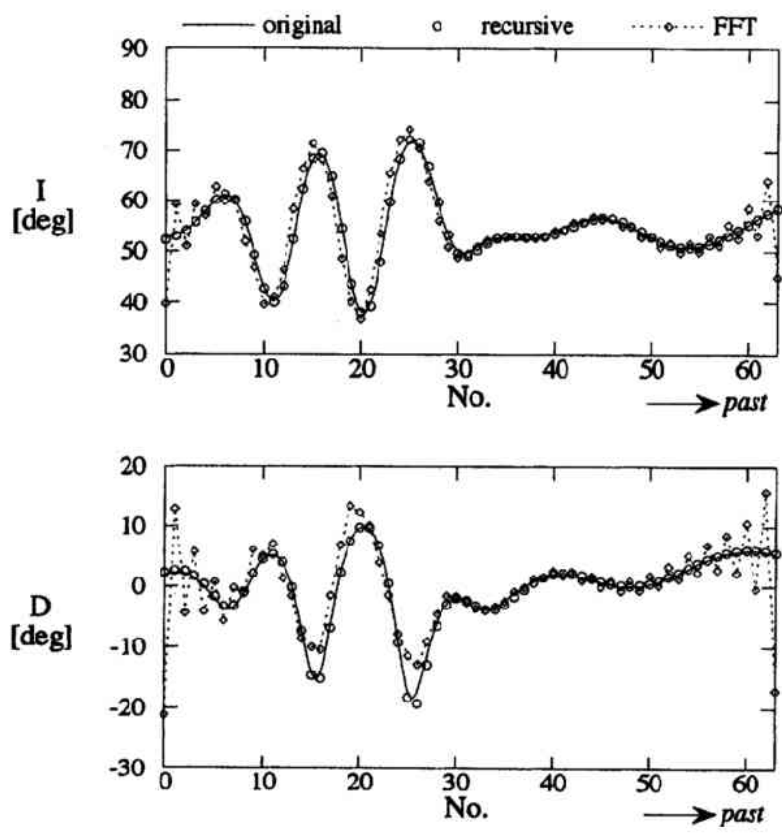

Fig. 4. Deconvolution results for the data set $G_{11}$ with the recursive method assuming the almost constant intensity of the convolved field and the known fixing factor $\left(A_{11}=0.2\right)$. The results with the FFT method are also shown. 


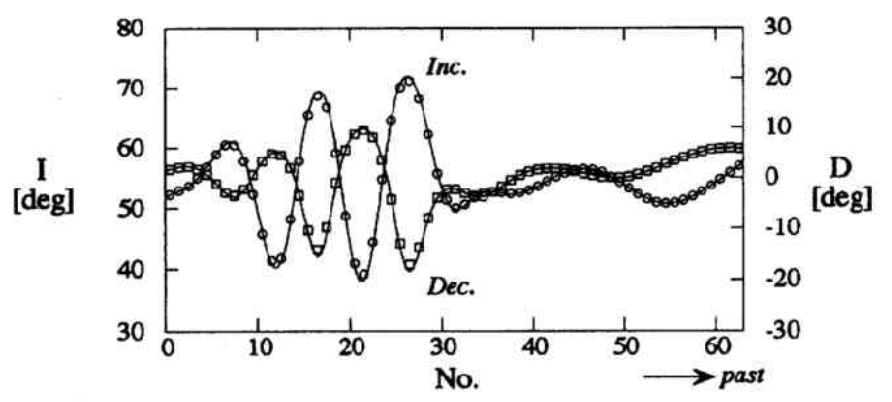

Fig. 5.

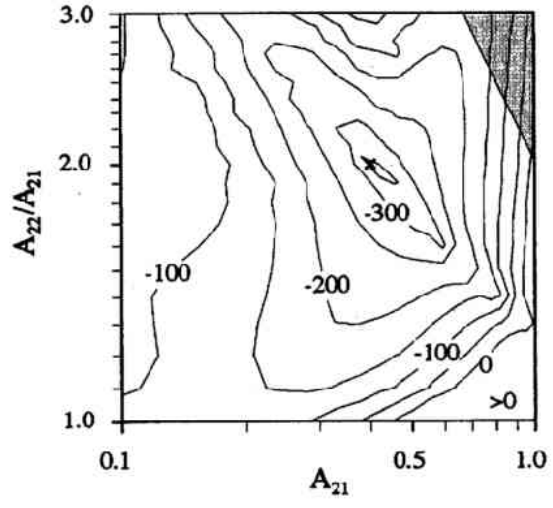

Fig. 6.

Fig. 5. Deconvolution results for the data sets of $G_{11}$ and $G_{12}$ with the recursive method assuming the almost constant intensity of the convolved field and the unknown fixing factors. From the least square fitting, the fixing factors are estimated to be of $A_{11}=0.205$ and $A_{12}=0.128$. Circles: inclination, and squares: declination.

Fig. 6. A contour map of $\Theta$ for the deconvolution of $G_{21}$ and $G_{22}$. The convergence point is of $A_{21}=0.40$ and $A_{22} / A_{21}=2.00$ with $\Theta=-360.0$. Cross: convergence point, hatched area: no meaningful solution because of $A_{22}>2$.
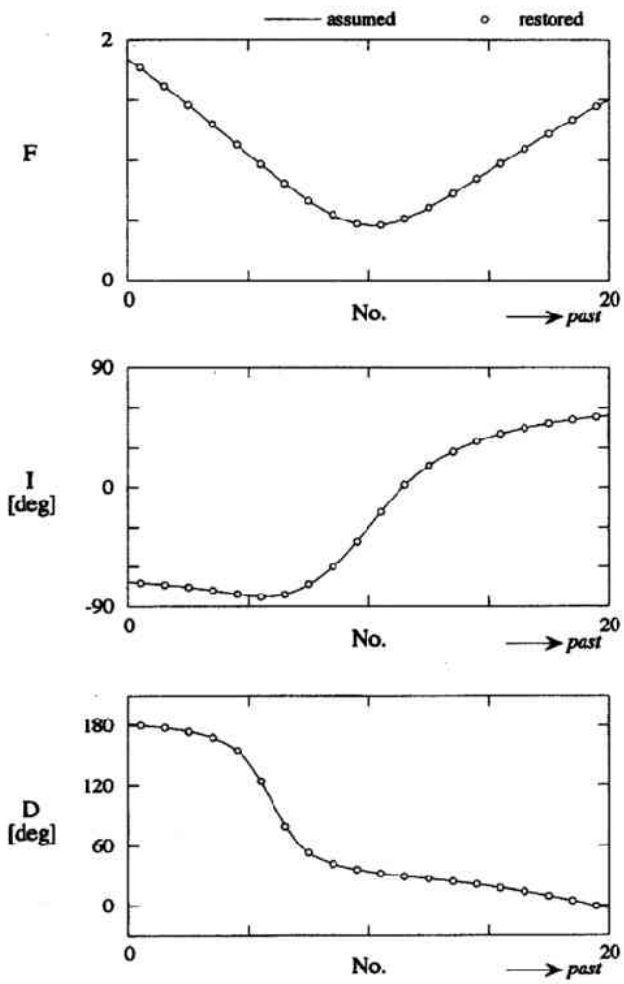

Fig. 7. Deconvolution results for the data sets $G_{21}$ and $G_{22}$ which contain no noises (sec Fig. 2). Three components of the original field are accurately restored from the directions (without intensities) of the $G_{21}$ and $G_{22}$ sets by the present deconvolution. Circles: restored components, and solid line: the assumed ones. 


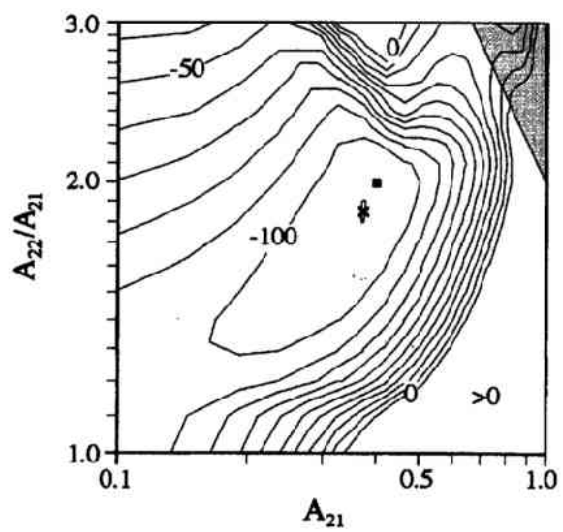

Fig. 8. A contour map of $\Theta$ for the deconvolution of the smoothed $G_{21}^{\prime}$ and $G_{22}{ }^{\prime}$. The convergence point is of $A_{21}=0.37$ and $A_{22} /$ $A_{21}=1.85$ with $\Theta=-110.2$. The square denotes the assumed point and other symbols are the same as in Fig. 6.
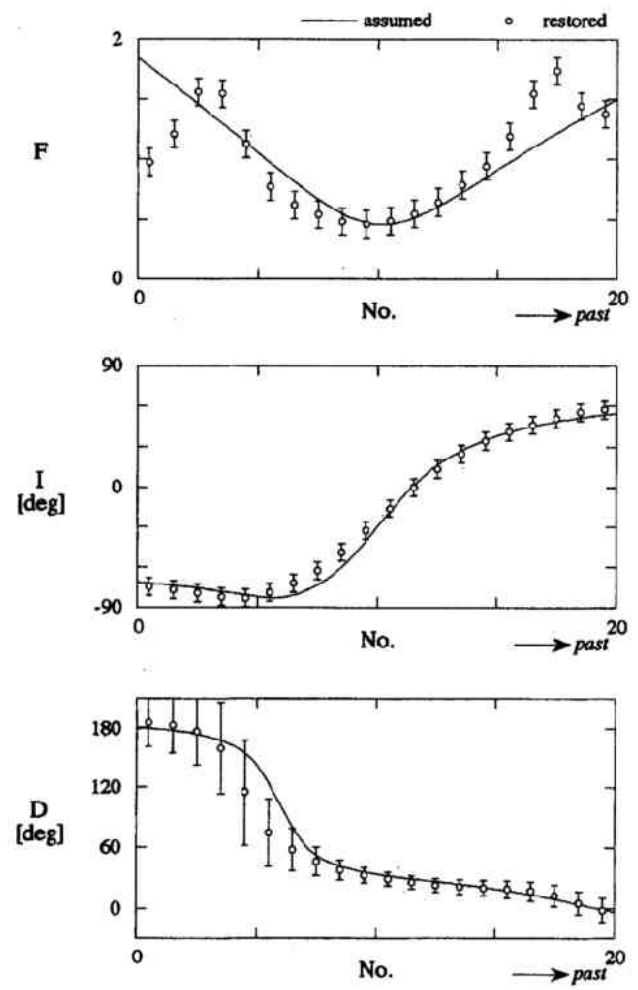

Fig. 9. Deconvolution results for the smoothed $G_{21}$ ' and $G_{22}{ }^{\prime}$ (see Fig. 3). The error bar is at a $95 \%$ confidence level. The symbols are the same as in Fig. 8.

$A_{1}=0.40, A_{22} / A_{21}=2.00$ and thus $A_{22}=0.80$, which are the same as the assumed values. Besides the calculated fields accurately follow the original ones (Fig. 7). Hence the present method works well in the condition of a variable intensity of the convolved field. 


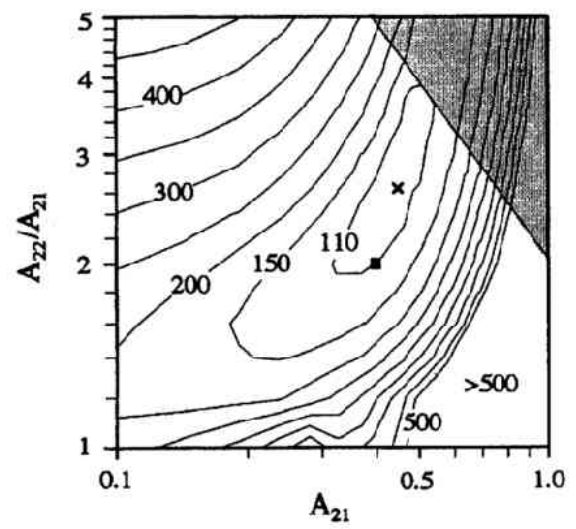

Fig. 10. A contour map of ABIC for the deconvolution of the smoothed $G_{21}{ }^{\prime}$ and $G_{22}{ }^{\prime}$. The convergence point is of $A_{21}=0.45$ and $A_{22} / A_{21}=2.60$ with $\mathrm{ABIC}=100.7$. The symbols are the same as in Fig. 7 .
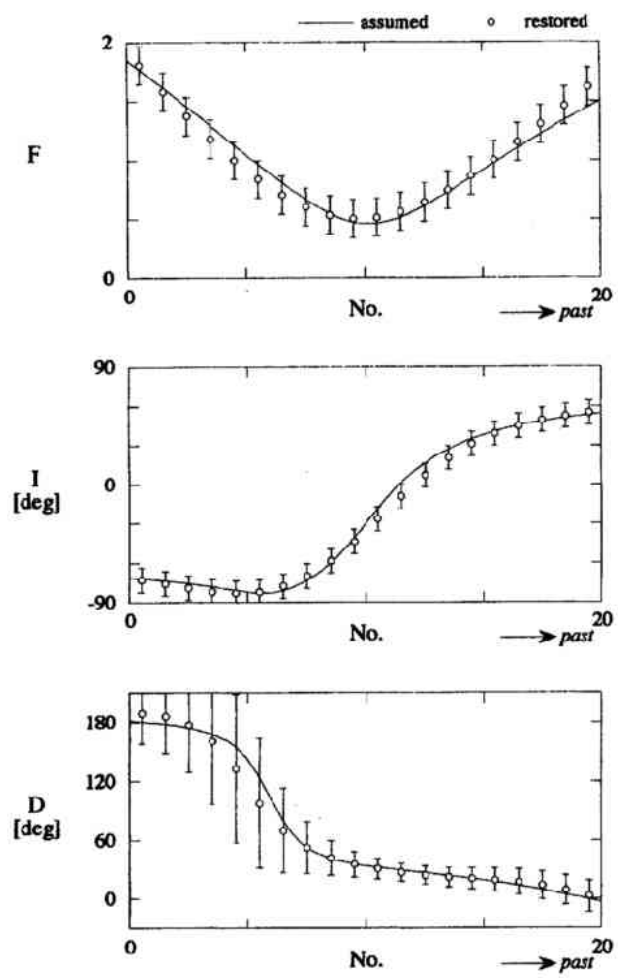

Fig. 11. Deconvolution results for the smoothed $G_{21}{ }^{\prime}$ and $G_{22}{ }^{\prime}$ considering the smoothness of the intensity of the convolved field. The error bar is at a $95 \%$ confidence level. The symbols are the same as in Fig. 7.

4.4 Two sites with variable $M_{i, j}$ different fixing factors and random noises $\left(G_{21}{ }^{\prime}, G_{22}\right)$

Applying the technique by Tsunakawa (1992), the directions of $G_{21}{ }^{\prime}$ and $G_{22}$ are smoothed and the equal-interval data are provided (Fig. 3). The random noises in those data sets are regarded as the instrumental, the magnetization instability, and so on. These noises are considered to be random at the 


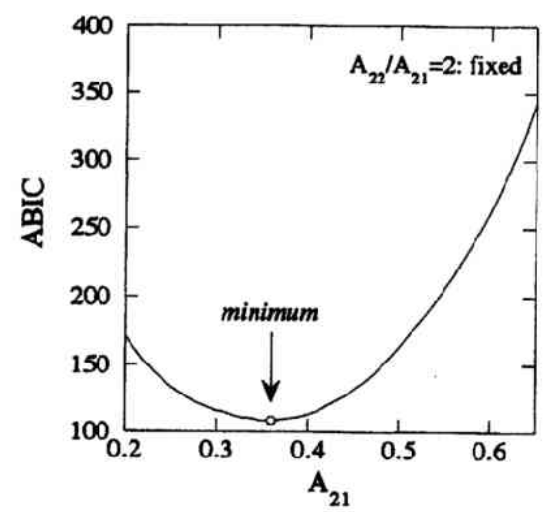

Fig. 12. ABIC change against $A_{21}$ in the condition of $A_{22} / A_{21}=2$ for the deconvolution of the smoothed $G_{21}{ }^{\prime}$ and $G_{22}{ }^{\prime}$. The ABIC minimum is of $A_{21}=0.36\left(A_{22}=0.72\right)$ with $\mathrm{ABIC}=107.8$.
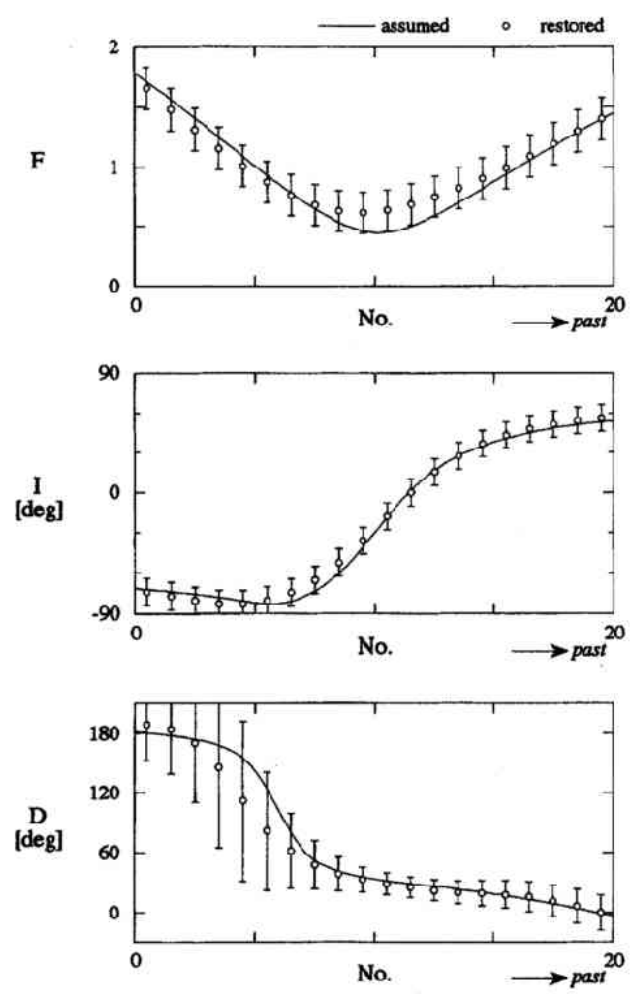

Fig. 13. Deconvolution results for the smoothed $G_{21}{ }^{\prime}$ and $G_{22}$ ' considering the smoothness of the intensity of the convolved field in the condition of $A_{22} / A_{21}=2$. The error bar is at a $95 \%$ confidence level. The symbols are the same as in Fig. 7.

measurement, so that they should be removed before deconvolution. The deconvolution cannot be accomplished without this smoothing in practice, because the solution is sensitive to noises included in the data as discussed later. On the other hand, the random fluctuation of the original field should be treated in the deconvolution process as $\varepsilon_{k l, j}$ in Eq. (35).

First, the minimization of $\Theta$ is applied to the smoothing results and then the convergence point is 
searched in the contour map (Fig. 8). The concave feature is again found but its convergence degree $(\Theta$ $=-110.2)$ becomes worth than in Fig. $6(\Theta=-360.0)$. The estimates of fixing factors are of $A_{21}=0.37$, $A_{22} / A_{21}=1.85$ and thus $A_{22}=0.69$, which are not far from the assumed ones. However, the deconvolved fields, in particular the relative intensities, are significantly scattering near both edges (Fig. 9). The deviation around the edges in Fig. 9 is probably caused by the small noises remaining after the smoothing. The estimates of inclinations and declinations seem less sensitive to the noise than relative intensities and fixing factors.

ABIC calculation was performed by applying Eq. (52). The calculation of ABIC yields the local minimum $(\mathrm{ABIC}=100.7)$ at a point of $A_{21}=0.45, A_{22} / A_{21}=2.60$ and $A_{22}=1.17$, though the contour pattern seems a trough (Fig. 10). Compared with the assumed values of the fixing factors, these estimates are 10 $40 \%$ larger. This may be again due to the noise remaining in the analysed data. The deconvolution is substantially based on the subtraction between the adjacent data, amplifying an effect of the noise on the relative intensity. This amplification is severe for a small fixing factor while the present deconvolution requires the smoothness of the relative intensity. As a result, fixing factors may be estimated to be larger.

The total intensity, inclination, and declination are shown in Fig. 11. These follow the assumed curves well though the slight shift is seen in part.

Suppose that the sedimentary material is homogeneous and that the fixing factors are determined from the sedimentation rate as in Eq. (64). For the data sets $G_{21}{ }^{\prime}$ and $G_{22}{ }^{\prime}$, the relationship of $A_{22} / A_{21}=$ 2 is assumed, that is, $z_{22} / z_{21}=2$. ABIC is shown against $A_{21}$ values in Fig. 12 and minimized at $A_{21}=0.36$ $\left(A_{22}=0.72\right)$. These estimates of the fixing factors are much better than in the condition that both of $A_{21}$ and $A_{22}$ are free. The intensity and direction changes are also restored well as in Fig. 13.

It is demonstrated that three components of the original field can be restored from the directions of the post-depositional DRM with the present method. However, the estimates of the relative intensities and fixing factors appear less accurate than the directional components. Taking some reasonable assumptions of fixing factors improves this situation to give more plausible values of parameters in deconvolution.

\section{Conclusions}

The new deconvolution method is proposed for the post-depositional DRM direction which is regarded as the convolution of the geomagnetic field. The response function is phenomenologically and theoretically approximated to be exponential in the first order. Using this function, the original field is expressed by the simple equation of the convolved field. The deconvolution method is formulated and examined in several cases using the synthetic data. When the convolved field has almost constant intensities, the deconvolution is very simple. Three components of the original field, including the relative intensity, are restored if multiple data sets of directions with different fixing factors are available.

The deconvolution can adequately restore the original field if the analysed data contain no noise. Noises in direction data affect estimates of fixing factors and relative intensities whereas directions seem to be restored well. However the assumption of the continuous change in the geomagnetic field leads us to extraction of signals by the Bayesian procedures in the smoothing and the deconvolution. Another reasonable constraint such as the relationship between fixing factors and sedimentation rates may reduce the noise effect on the deconvolution results.

The author is grateful to Dr. H. Shibuya for his critical and constructive comments. Dr. M. Hyodo is also thanked for his helpful review. This study was partly supported by the Grant-in-Aid from Ministry of Education, Science, and Culture of Japan.

\section{APPENDIX A}

Equation (26) is rewritten to be: 


$$
M_{i}(t) \boldsymbol{m}_{i}(t)=\int_{0}^{t} \boldsymbol{f}(\tau) A_{i} \exp \left\{A_{i}(\tau-t)\right\} d \tau
$$

Integrating both sides of Eq. (A1) for $\theta_{1} \leq t \leq \theta_{2}$,

$$
\int_{\theta_{1}}^{\theta_{2}} M_{i}(t) \boldsymbol{m}_{i}(t) d t=\int_{\theta_{1}}^{\theta_{2}} d t \int_{0}^{t} f(\tau) A_{i} \exp \left[A_{i}(\tau-t)\right] d t
$$

We consider the domain of $D_{\eta}: \tau \leq t \leq \eta, 0 \leq \tau \leq \eta$.

$$
\begin{aligned}
& \iint_{D_{\eta}} d t d \tau \boldsymbol{f}(\tau) A_{i} \exp \left\{A_{i}(\tau-t)\right\} \\
& =\int_{0}^{\eta} d \tau \boldsymbol{f}(\tau) A_{i} \int_{\tau}^{\eta} \exp \left\{A_{i}(\tau-t)\right\} d t \\
& =\int_{0}^{\eta} \boldsymbol{f}(\tau)\left[1-\exp \left\{A_{i}(\tau-\eta)\right\}\right] d \tau .
\end{aligned}
$$

Therefore,

$$
\begin{aligned}
& \int_{\theta_{1}}^{\theta_{2}} d t \int_{0}^{t} \boldsymbol{f}(\tau) A_{i} \exp \left[A_{i}(\tau-t)\right] d t \\
& =\iint_{D_{\theta_{2}}} d t d \tau \boldsymbol{f}(\tau) A_{i} \exp \left\{A_{i}(\tau-t)\right\}-\iint_{D_{\theta_{1}}} d t d \tau \boldsymbol{f}(\tau) A_{i} \exp \left\{A_{i}(\tau-t)\right\} \\
& =\int_{0}^{\theta_{2}} \boldsymbol{f}(\tau)\left[1-\exp \left\{A_{i}\left(\tau-\theta_{2}\right)\right\}\right] d \tau-\int_{0}^{\theta_{1}} \boldsymbol{f}(\tau)\left[1-\exp \left\{A_{i}\left(\tau-\theta_{1}\right)\right\}\right] d \tau \\
& =\int_{\theta_{1}}^{\theta_{2}} \boldsymbol{f}(\tau) d \tau-\int_{0}^{\theta_{2}} \boldsymbol{f}(\tau) \exp \left\{A_{i}\left(\tau-\theta_{2}\right)\right\} d \tau-\int_{0}^{\theta_{1}} \boldsymbol{f}(\tau) \exp \left\{A_{i}\left(\tau-\theta_{1}\right)\right\} d \tau \\
& =\int_{\theta_{1}}^{\theta_{2}} \boldsymbol{f}(\tau) d \tau+\left\{M_{i}\left(\theta_{1}\right) \boldsymbol{m}_{i}\left(\theta_{1}\right)-M_{i}\left(\theta_{2}\right) \boldsymbol{m}_{i}\left(\theta_{2}\right)\right\} / A_{i} .
\end{aligned}
$$

This relationship gives the next equation.

$$
\int_{\theta_{1}}^{\theta_{2}} M_{i}(t) \boldsymbol{m}_{i}(t) d t=\int_{\theta_{1}}^{\theta_{2}} \boldsymbol{f}(\tau) d \tau+\left\{M_{i}\left(\theta_{1}\right) \boldsymbol{m}_{i}\left(\theta_{1}\right)-M_{i}\left(\theta_{2}\right) \boldsymbol{m}_{i}\left(\theta_{2}\right)\right\} / A_{i} .
$$

The averaged field vector of $f(t)$ is defined as

$$
\bar{f}\left(\theta_{1}, \theta_{2}\right)=\frac{1}{\theta_{2}-\theta_{1}} \int_{\theta_{1}}^{\theta_{2}} \boldsymbol{f}(\tau) d \tau
$$

This is transformed into

$$
\left(\theta_{2}-\theta_{1}\right) \overline{\boldsymbol{f}}\left(\theta_{1}, \theta_{2}\right)=\int_{\theta_{1}}^{\theta_{2}} M_{i}(t) \boldsymbol{m}_{i}(t) d t-\left\{M_{i}\left(\theta_{1}\right) \boldsymbol{m}_{i}\left(\theta_{1}\right)-M_{i}\left(\theta_{2}\right) \boldsymbol{m}_{i}\left(\theta_{2}\right)\right\} / A_{i}
$$

$\theta_{1}$ and $\theta_{2}$ are replaced by $t_{j}$ and $t_{j+1}$, respectively, and then the first integration of the right-hand side is 
approximated with the trapezoidal rule as follows.

$$
\int_{t_{j}}^{t_{j+1}} M_{i}(t) \boldsymbol{m}_{i}(t) d t=\left(M_{i, j} \boldsymbol{m}_{i, j}+M_{i, j+1} \boldsymbol{m}_{i, j+1}\right) \Delta t / 2
$$

Consequently Eqs. (27)-(29) are deduced from Eqs. (A7) and (A8).

APPENDIX B

$$
V=\left[\begin{array}{cccccc}
V_{1} & -V_{2} & o & & \cdots & o \\
V_{1} & o & -V_{3} & o & \cdots & o \\
V_{1} & o & \cdots & \cdots & o & -V_{N} \\
o & V_{2} & -V_{3} & o & \cdots & o \\
o & V_{2} & o & \cdots & o & -V_{N} \\
o & & \cdots & o & V_{N-1} & -V_{N}
\end{array}\right]
$$

where

$$
V_{k}=\left[\begin{array}{c}
V_{x k} \\
V_{y k} \\
V_{z k}
\end{array}\right]
$$

and

$$
V_{\alpha k}=\left[\begin{array}{cccccc}
c_{k}^{-} m_{\alpha 1,1} & c_{k}^{+} m_{\alpha 1,2} & 0 & & \cdots & 0 \\
0 & c_{k}^{-} m_{\alpha 1,2} & c_{k}^{+} m_{\alpha 1,3} & 0 & \cdots & 0 \\
& & \cdots & & & \\
0 & \cdots & & 0 & c_{k}^{-} m_{\alpha 1, n-1} & c_{k}^{+} m_{\alpha 1, n}
\end{array}\right]
$$

where $\alpha=x, y$ and $z$. $O$ denotes the zero matrix.

$$
\text { APPENDIX C }
$$

$$
W=\left[\begin{array}{l}
V \\
U
\end{array}\right],
$$

where

$$
U=\left[\begin{array}{cccc}
u_{1} D & O & O & O \\
O & u_{2} D & O & O \\
& & \cdots & \\
O & O & O & u_{N} D
\end{array}\right]
$$


$D$ denotes the following matrix,

$$
D=\left[\begin{array}{cccccccc}
\beta & 0 & & & & & & 0 \\
-2 \beta & \beta & 0 & & & & & 0 \\
1 & -2 & 1 & 0 & \cdots & & & 0 \\
0 & 1 & -2 & 1 & 0 & \cdots & & 0 \\
& & & \cdots & & & & \\
& & & & & & & \\
0 & & \cdots & 0 & 1 & -2 & 1 & 0 \\
0 & & & \cdots & 0 & 1 & -2 & 1
\end{array}\right]
$$

for the second order. $\beta$ is a constant to reduce the edge effect (Akaike, 1980). Its value is tentatively of $10^{-4}$. This assumption does not affect ABIC if its value is the same through the analysis.

\section{APPENDIX D}

The integration term in Eq. (57) cannot analytically been solved because $\Sigma_{F}$ is a function of $\Lambda$. However, compared with the exponential term of $S_{b}, \Sigma_{F}$ is moderately changed in the vicinity of the optimal solution $\Lambda_{1}$. This is because $\Sigma_{F}$ is a sum of $F_{j}^{-2}$ while the other term is an exponential function of subtraction of $\boldsymbol{F}_{j}$. Therefore $\Sigma_{F}$ is approximated to be $\Sigma_{F}{ }^{*}$ calculated from $\Lambda_{1}$.

Considering the coordinate system $\left\{p_{i}\right\}$ on the basis of the eigenvectors of $W^{\mathrm{T}} W$,

$$
\begin{aligned}
& \int_{|\mathbf{\Lambda}|=1} \Sigma_{F}^{-3(n-1) / 2} \exp \left[-3 S_{b} / 2 \sigma^{2}\right] d \mathbf{\Lambda} \\
& =\left(\Sigma_{F}^{*}\right)^{-3(n-1) / 2} \int_{\sum_{i=1}^{2 n} p_{i}^{2}=1} \exp \left[-3\left(\sum_{i=1}^{2 n} \mu_{i} p_{i}^{2}\right) / 2 \sigma^{2}\right] d p_{1} \ldots d p_{2 n} \\
& =\left(\Sigma_{F}^{*}\right)^{-3(n-1) / 2} \exp \left(-3 \mu_{1} / 2 \sigma^{2}\right) \int_{\sum_{i=1}^{2 n} p_{i}^{2}=1} \exp \left[-3\left\{\mu_{1}\left(p_{1}^{2}-1\right)+\sum_{i=2}^{2 n} \mu_{i} p_{i}^{2}\right\} / 2 \sigma^{2}\right] d p_{1} \ldots d p_{2 n} \\
& =\left(\Sigma_{F}^{*}\right)^{-3(n-1) / 2} \exp \left(-3 \mu_{1} / 2 \sigma^{2}\right) \int_{\sum_{i=2}^{2 n} p_{i}^{2} \leq 1} \exp \left[-3\left\{\mu_{1}\left(-\sum_{i=2}^{2 n} p_{i}^{2}\right)+\sum_{i=2}^{2 n} \mu_{i} p_{i}^{2}\right\} / 2 \sigma^{2}\right] d p_{2} \ldots d p_{2 n} \\
& =\left(\Sigma_{F}^{*}\right)^{-3(n-1) / 2} \exp \left(-3 \mu_{1} / 2 \sigma^{2}\right) \int_{\sum_{i=2}^{2 n} p_{i}^{2} \leq 1} \exp \left[-3\left\{\sum_{i=2}^{2 n}\left(\mu_{i}-\mu_{1}\right) p_{i}^{2}\right\} / 2 \sigma^{2}\right] d p_{2} \ldots d p_{2 n} .
\end{aligned}
$$

Usually $\mu_{1}<<\mu_{i}(i \geq 2)$ and then,

$$
\begin{aligned}
& \int_{|\Lambda|=1} \Sigma_{F}^{-3(n-1) / 2} \exp \left[-3 S_{b} / 2 \sigma^{2}\right] d \Lambda \\
& \approx\left(\Sigma_{F}^{*}\right)^{-3(n-1) / 2} \exp \left(-3 \mu_{1} / 2 \sigma^{2}\right) \int_{-\infty}^{+\infty} \exp \left[-3\left\{\sum_{i=2}^{2 n}\left(\mu_{i}-\mu_{1}\right) p_{i}^{2}\right\} / 2 \sigma^{2}\right] d p_{2} \ldots d p_{2 n} \\
& \approx\left(\Sigma_{F}^{*}\right)^{-3(n-1) / 2} \exp \left(-3 \mu_{1} / 2 \sigma^{2}\right) \prod_{i=2}^{2 n}\left\{2 \pi \sigma^{2} / 3\left(\mu_{i}-\mu_{1}\right)\right\}^{1 / 2} .
\end{aligned}
$$




\section{APPENDIX E}

The optimal solutions of the components $X_{j}{ }^{*}, Y_{j}{ }^{*}$ and $Z_{j}{ }^{*}$, the total intensity $F_{j}{ }^{*}$, horizontal component $H_{j}^{*}$, inclination $I_{j}^{*}$, and declination $D_{j}^{*}$ are defined as follows.

$$
\begin{aligned}
& X_{j}^{*}=g_{x j}^{*}, \\
& Y_{j}^{*}=g_{y j}^{*}, \\
& Z_{j}^{*}=g_{z j}^{*}, \\
& F_{j}^{*}=\left(g_{x j}^{* 2}+g_{y j}^{* 2}++_{z j}^{* 2}\right)^{1 / 2}, \\
& I_{j}^{*}=\sin ^{-1}\left(g_{z j}^{*} / F_{j}^{*}\right), \\
& D_{j}^{*}=\tan ^{-1}\left(g_{y j}^{*} / g_{x j}^{*}\right) .
\end{aligned}
$$

Their $95 \%$ coincidence levels are as below.

$$
\begin{aligned}
& \Delta_{95} F_{j}^{*}=1.96 \sigma^{*}, \\
& \Delta_{95} X_{j}^{*}=\Delta_{95} Y_{j}^{*}=\Delta_{95} Z_{j}^{*}=1.96 \sigma^{*} / \sqrt{3}, \\
& \Delta_{95} I_{j}^{*}=159 / \sqrt{\kappa_{0}}[\operatorname{deg}] \\
& \Delta_{95} D_{j}^{*}=\Delta_{95} I_{j}^{*} / \cos I_{j}^{*}[\mathrm{deg}]
\end{aligned}
$$

where

$$
\kappa_{0}=2 / \sigma^{* 2}
$$

\section{REFERENCES}

Akaike, H., Likelihood and the Bayes procedure, in Bayesian Statistics, edited by J. M. Bemardo, M. H. DeGroot, D. V. Lindley, and A. F. M. Smith, pp. 143-166, University Press, Valencia, Spain, 1980.

Anson, G. L. and K. P. Kodama, Compaction-induced shallowing of the post-depositional remanent magnetization in a synthetic sediment, Geophys. J. R. astr. Soc., 88, 673-692, 1987.

Arason, P. and S. Levi, Compaction and Inclination Shallowing in deep-sea sediments from the Pacific Ocean, J. Geophys. Res., 95, 4501-4510, 1990.

Barton, C. E. and M. W. McElhinny, Detrital remanent magnetization in five slowly redeposited long cores of sediments, Geophys. Res. Lett., 6, 229-232, 1979.

Barton, C. E., M. W. McElhinny, and D. J. Edwards, Laboratory studies of depositional DRM, Geophys. J. R. astr. Soc., 61, 355$377,1980$.

Blow, R. A. and N. Hamilton, Effect of compaction on the acquisition of a detrital remanent magnetization in fine grained sediments, Geophys. J. R. astr. Soc., 52, 13-23, 1978. 
Deamer, G. A. and K. P. Kodama, Compaction-induced inclination shallowing in synthetic and natural clay-rich sediments, $J$. Geophys. Res., 95, 4511-4529, 1990.

Fletcher, R. and M. J. D. Powell, A rapid convergent descent method for minimization, The Computer J., 6, 163-168, 1963.

Guinasso, N. L., Jr. and D. R. Schink, Quantitative estimates of biological mixing rates in abyssal sediments, J. Geophys. Res., 80, 3032-3043, 1975.

Hamano, Y., An experiment on the post-depositional remanent magnetization in artificial and natural sediments, Earth Planet. Sci. Lett., 51, 221-232, 1980.

Hyodo, M., Possibility of reconstruction of the past geomagnetic field from homogeneous sediments, J. Geomag. Geoelectr., 36, 45-62, 1984.

Hyodo, M., C. Itota, and K. Yaskawa, Geomagnetic secular variation reconstructed from magnetizations of wide-diameter cores of Holocene sediments in Japan, J. Geomag. Geoelectr., 45, 669-696, 1993.

Irving, E., Origin of the paleomagnetism of the Torridonian sandstones of north-west Scotland, Phil. Trans. Roy. Soc. London, Ser. A, 250, 100-110, 1957.

Irving, E. and A. Major, Post-depositional detrital remanent magnetization in a synthetic sediment, Sedimentology, 3, 135-143, 1964.

Jaeger, J. C. and N. G. W. Cook, Fundamentals of Rock Mechanics, Third ed., 593 pp., Chapmann and Hall, London, 1979.

Kent, D. V., Post-depositional remanent magnetization in a deep-sea sediment, Nature, 246, 32-34, 1973.

King, R.F., Remanent magnetism of artificially deposited sediments, Mon. Notic. Roy. Astron. Soc. Gephys. Suppl., 7, 115-134, 1955.

Løvlie, R., Post-depositional remanent magnetization in a redeposited deep-sea sediment, Earth Planet. Sci. Lett., 21, 315-320, 1974.

Løvlie, R., The intensity pattern of post-depositional remanence acquired in some marine sediments during a reversal of the external magnetic field, Earth Planet. Sci. Lett., 30, 209-214, 1976.

Nagata, T., Notes on detrital remanent magnetization of sediments, J. Geomag. Geoelectr., 14, 99-106, 1962.

Niitsuma, N., Zone-magnetization model and depth lag of NRM in deep-sea sediments, Rock Magn. Paleogeophys., 4, 65-71, 1977.

Opdyke, N.D., D. V. Kent, and W. Lowrie, Details of magnetic polarity transitions recorded in a high deposition rate, Earth Planet. Sci. Lett., 20, 315-324, 1973.

Otofuji, Y. and S. Sasajima, A magnetization process of sediments: laboratory experiments on post-depositional remanent magnetization, Geophys. J. R. astr. Soc., 66, 241-259, 1981

Payne, M. A. and K. L. Verosub, The acquisition of post depositional detrital remanent magnetization in a variety of natural sediments, Geophys. J. R. astr. Soc., 68, 625-642, 1982.

Raisbeck, G. M., F. Yiou, D. Bourles, and D. V. Kent, Evidence for an increase in cosmogenic ${ }^{10}$ Be during a geomagnetic reversal, Nature, 315, 315-317, 1985.

Tsunakawa, H., Bayesian approach to smoothing the palaeomagnetic data using ABIC, Geophys. J. Int., 108, 801-811, 1992.

Verosub, K. L., Depositional and post-depositional processes in the magnetization of sediments, Rev. Geophys., 15, 129-143, 1977.

Verosub, K. L., R. A. Ensley, and J. S. Ulrick, The role of water content in the magnetization of sediments, Geophys. Res. Lett., 6, 226-228, 1979.

Yamazaki, T., Fixation of remanent magnetization with compaction in deep-sea environment, J. Geomag. Geoelectr., 39, 307$312,1987$.

Yaskawa, K., When did magnetization fix in loose sediments?, Rock Magn. Paleogeophys., 2, 15-19, 1974. 